\title{
The birth of microsatellites
}

SIR - Microsatellites, very short ( $2-5$ basepair) tandem nucleotide repeats, are found scattered throughout the genomes of eukaryotes $^{1,2}$. Those loci with a high number of repeat units tend to vary in repeat number among individuals in a species, making them useful in many types of modern genetic studies ${ }^{3}$. Microsatellites have gained notoriety through their association with certain genetic diseases in humans ${ }^{4-6}$. Much has been written about the probable mechanisms causing length variation within species $^{2,6,7}$, but little is known about the evolutionary origins of microsatellites ${ }^{1}$. Here we document the independent 'birth' of two separate microsatellites in a short region of the $\eta$-globin pseudogene in primates.

As shown in the figure, in many species of primates the DNA sequence in this region is a single nucleotide substitution away from the creation of two different types of simple repeat sequences. Within the hominoids, a single nucleotide substitution of a $\mathrm{G}$ to an A occurred along the lineage leading to the common ancestor of the African apes (gorilla, bonobo and chimpanzee) and humans. This substitution created a tetranucleotide repeat, $(\text { ATGT })_{2}$, which is expanded to (ATGT) $)_{4}$ in the African apes and to (ATGT) $)_{5}$ in humans. As only one sequence is available from each species, we do not know if intraspecific variation in repeat number exists at this locus. However, such polymorphism is unlikely, because loci with fewer than about 5 to 8 repeat units are almost never polymorphic in humans ${ }^{1,8}$.
A separate nucleotide substitution occurred within the New World monkeys along the lineage leading to owl monkey. This A-to-G transition created a dinucleotide repeat, $(\mathrm{GT})_{5}$ or $(\mathrm{TG})_{6}$, which has expanded by one repeat unit in this species. $\eta$-Globins from other New World monkeys that are more closely related to this owl monkey need to be sequenced to trace the evolution of this new microsatellite more completely, especially to look for additional expansions.

Even though a wide phylogenetic spread of primates has three contiguous GTs and four contiguous TGs in this region of the $\eta$-globin pseudogene, of the species sampled, only the owl monkey has a dinucleotide expansion. Thus, a minimum number of repeat units may be necessary before initial expansion occurs. In this dinucleotide repeat case, the minimum number appears to be four or five, and in the tetranucleotide repeat case, two. The evolutionary coupling of the nucleotide substitutions and the expansions suggests that the point mutations created enough repeat units for subsequent microsatellite expansion. The expansions we have found in this region of the $\eta$-globin gene are consistent with a stepwise mutation model for microsatellite evolution ${ }^{1,2}$ in which short arrays increase or decrease in repeat number by one or two repeats, probably by replication slippage. We propose that initial expansion of short repeat sequences, such as we document here, occurs relatively slowly on an evolutionary timescale.
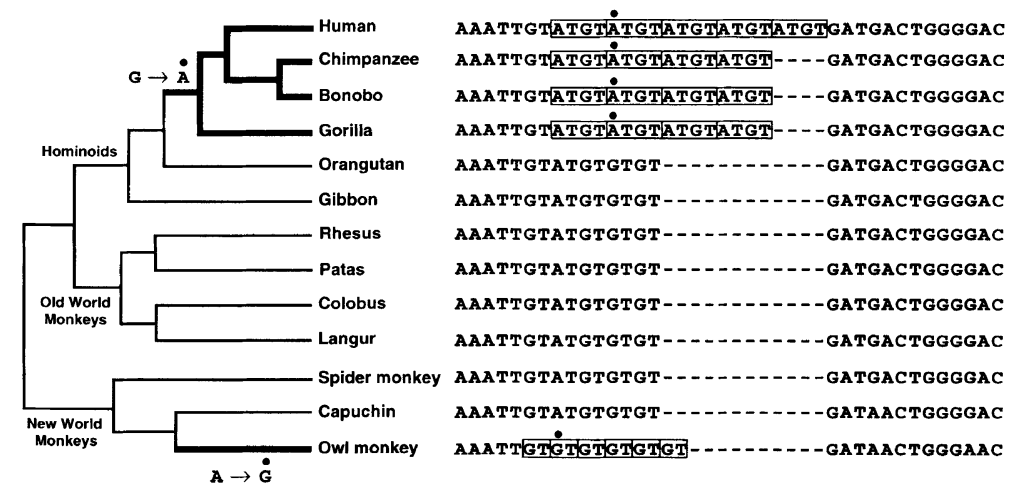

A short portion of a longer alignment of all available nucleotide sequences of the $\eta$-globin pseudogene of primates. The multiple alignment of these primate sequences is unambiguous surrounding this region (which encompasses nucleotides 1,311-1,350 of the full-length human sequence, and would be in the second intron of an expressed globin gene). Gaps relative to the human sequence are indicated by dashes. Those nucleotide substitutions (indicated by dots) that created repeat units are mapped onto the known phylogeny of these primates in the manner that requires the fewest events. Boxes delineate microsatellite repeats. For the sake of clean alignment to the human sequence, we have boxed the GT repeats in the owl monkey; however, it is just as likely that this expansion involved a TG repeat, as mutations in the hominoids should have no effect on those in the New World monkeys. GenBank accession numbers: Human, X02133; chimpanzee, K02542; bonobo, X79724; gorilla, K02543; orangutan, M18038; gibbon, M54985; owl monkey, X02142; spider monkey, J03050; white-fronted capuchin, M81409. Rhesus macaque sequence is from ref. 9. Sequences from patas monkey, Angolan colobus and Hanuman langur were generated in our laboratory by P. Banerjee; analysis of these pseudogenes will be published elsewhere.
Once a critical number of repeat units ${ }^{1,8}$ has arisen in a given species, that locus can become hypervariable, with mutations occurring even on a generational timescale $^{5}$. A broader understanding of microsatellite birth and early evolution will require the sequencing of regions surrounding other expanded loci and analysis of these data in proper phylogenetic context.

\section{Walter Messier \\ Shou-Hsien Li \\ Caro-Beth Stewart}

Department of Biological Sciences,

University at Albany,

State University of New York,

Albany, New York 12222, USA

1. Valdes, A. M., Slatkin, M. \& Freimer, N. B. Genetics 133 737-748 (1993).

2. Charlesworth, B. et al. Nature 371, 215-220 (1994).

3. Dowling, T. E. et al. in Molecular Systematics (eds Hillis, D. M. et al.) Ch. 8 (Sinauer, Sunderland, MA, 1996).

4. Burke, J. R. et al. Nature Med. 2, 347-350 (1996).

5. Campuzano, V. et al. Science 271, 1423-1427 (1996).

6. Kuhl, D. P. A. \& Caskey, C. T. Curr. Opin. Genet. Dev. 3, 404-407 (1993).

7. Rubinsztein, D. C. et al. Nature Genet. 10, 337-343 (1995)

8. Armour, J. A. L. et al. Hum. molec. Genet. 3, 599-605 (1994).

9. Koop, B. F. et al. Nature 319, 234-238 (1986)

\section{Cosmological constant}

SIR - Taken in sum ${ }^{1}$, an impressive variety of observations indicate that the cosmological constant $(\Lambda)$ is not zero. Principal among these is the existence of stars that are more than 15 billion years $\mathrm{old}^{2}$, in a Universe which is apparently much younger (less than 12 billion years old), based on recent determinations of the Hubble constant ${ }^{3}$. A cosmological constant increases the age of the Universe, but only modestly in a flat Universe. Even with a cosmological constant of $\Omega_{\Lambda}=0.7$ (where $\Omega_{\Lambda}$ is expressed as a fraction of the critical density), concordance between these ages relies heavily on measurement errors ${ }^{2,3}$.

Moreover, the cosmological constant has a strong effect ${ }^{4}$ on the deceleration parameter $q_{0}$, which provides an independent test of concordance. In particular, $q_{0}=1 / 2 \Omega_{\mathrm{m}}-\Omega_{\Lambda}$, where $\Omega_{\mathrm{m}}$ is the density of matter as a fraction of the critical density. If $\Omega_{\mathrm{m}}=0.3$ and $\Omega_{\Lambda}=0.7$ as suggested ${ }^{1}$, then $q_{0}=-0.55$. Although presently fraught with uncertainty, attempts ${ }^{5}$ to measure $q_{0}$ directly usually suggest positive, rather than negative, values. If $q_{0} \geq 0$ in a flat Universe, $\Omega_{\Lambda} \leq 1 / 2 \Omega_{\mathrm{m}}$, and the cosmological constant cannot be significant.

\section{Stacy S. McGaugh}

Department of Terrestrial Magnetism, Carnegie Institution of Washington, Washington, DC 20015, USA

\footnotetext{
1. Ostriker, J. P. \& Steinhardt, P. J. Nature $\mathbf{3 7 7}, 600-602$ (1995)

2. Bolte, M. \& Hogan, C. J. Nature 376, 399-402 (1995)

3. Freedman, W. L. et al. Nature 371, 757-762 (1994).

4. Peebles, P. E. J. Principles of Physical Cosmology (Princeton Univ. Press, 1993).

5. Sandage, A. R. in The Deep Universe, Saas-Fee Advanced Course 23 (Springer, New York, 1995).
} 